\title{
Covid 19 y las repercusiones en la educación en Guatemala
}

\author{
Covid 19 and its repercussions \\ on the education in Guatemala
}

Recibido 25/09/2020

Carmen María Santiso Rodríguez

Doctorado en Educación

Universidad de San Carlos de Guatemala

carmenmasantiso@gmail.com

ORCIC 0000-0002-3508-0878

Aceptado 18/01/2021

\section{Referencia}

Santiso Rodríguez, C. M. (2021). Covid 19 y las repercusiones en la educación en Guatemala. Revista Docencia Universitaria, 2(1), 51-61. https://doi.org/10.46954/revistadusac.v2i1.24

\section{Resumen}

La educación es un factor importante para el desarrollo de cualquier país, ya que influye en el avance y progreso (Plan Educativo Nacional, 2020) desde el 2020 se atravieza la pandemia Covid 19. El Ministerio de Educación propuso e implementó estrategias educativas de acuerdo a las posibilidades de los establecimientos y de las familias de los estudiantes, muchos fueron a través de plataformas virtuales, y otros, por medio de chats y muchos más con guías de estudio para que los niños realizarán las tareas y aprendieran las lecciones y el mismo proceso se devolvían a los maestros para que tuvieran oportunidad de calificar.

Después de un proceso de investigación descriptiva con enfoque cualitativo, en donde se entrevistó a maestros y a directores, y se logró evidenciar que la educación a distancia no es adecuada para 
Palabras clave:

covid 19, educación, educación a distancia, competencias, estudiantes

Keywords:

covid 19, education, virtual-learning, competence, students. países en desarrollo como Guatemala, porque no se tiene el acceso a tecnología en todos los sectores de la población. Y que, debido a lo abrupto del cambio en el ciclo escolar 2019, el proceso de aprendizaje de los estudiantes y las competencias requeridas por el Ministerio de Educación, no fueron del todo logradas, de acuerdo a lo expresado por maestros y directores.

\section{Abstract}

Education is an important factor for the development of any country, as it influences advancement and progress (Plan Educativo Nacional, 2020) since 2020, the Covid 19 pandemic has been going through. The Ministry of Education proposed and implemented educational strategies according to the possibilities of the educational establishments and the families of the students. Some classes were given through virtual platforms, others, through chats, and many more through study guides so that students would be able to complete the assignments and learn the lessons and let teacher grade their students' work.

After a descriptive research process with a qualitative approach, where teachers and principals were interviewed, it was possible to show that distance education is not adequate for developing countries such as Guatemala because there is no enough access to technology for all sectors of the population. And that, due to the abrupt change in the 2019 school year, the learning process of the students and the competencies required by the Ministry of Education were not fully achieved, according to what was expressed by teachers and principals.

\section{Introducción}

El año 2020 cambio el rumbo de la historia a nivel mundial la pandemia SARS COV-2 denominada COVID-19 vino a revolucionar la forma de vida en todos los ambientes, el trabajo se convirtió en teletrabajo, la educación presencial se trasladó a distancia. (Organización Mundial de la Salud, 2020) y cada institución educativa en Guatemala estableció opciones de acuerdo a sus condiciones y a los recursos de las familias de los estudiantes.

Las restricciones que el gobierno de Guatemala impuso a partir de la primera semana de marzo de 2020, fue el confinamiento, obligando a un aislamiento social, pero con las obligaciones de continuar con 
educación en la modalidad a distancia (Ministerio de Educación, 2020). Este confinamiento que se dio a nivel nacional, afectó a todos los sectores, y, el sistema educativo buscó las opciones para continuar con la educación; fue allí en donde la educación a distancia comenzó en todos los niveles, y tomando por sorpresa a los maestros, alumnos y a los padres porque nadie estaba preparado para un cambio tan drástico (Sánchez-Villena, \& De La Fuente-Figuerola, 2020).

La educación en Guatemala regida por el Curriculum Nacional Base, la cual define las competencias que cada estudiante debe alcanzar de acuerdo al grado cursado. Y tratando de cubrir contenidos en el área de escuelas e institutos, otorgó la responsabilidad en los directores y maestros para crear canales de comunicación con los estudiantes y además, creó estrategias como guías de estudio para su desarrollo, clases por televisión para niños de primaria, mismas que fueron impartidas por maestros de grado, con la experiencia y vocabulario adecuado, tomando en cuenta que la transmisión era por los canales nacionales con el fin de llegar a cada lugar en donde hubiera un niño con el deseo de estudiar, por otro lado se publicaron por medio de los medios de comunicación lecciones que fortalecían el proceso académico, como ayuda adicional (Ola, 2020).

Los establecimientos educativos, tanto públicos como privados, buscaron formas de continuar con la educación utilizando para ello aplicaciones tecnológicas y recursos adicionales que contribuyen con la comunicación maestro y estudiantes, (Agenda Guatemalteca de Noticias, 2021), pero ¿esta comunicación a distancia fue suficiente para que los alumnos lograrán alcanzar las competencias educativas requeridas por el Curriculum Nacional Base y el Ministerio de Educación de acuerdo a los niveles estudiados?

Las instituciones privadas en su mayoría utilizaron aplicaciones para comunicarse con sus estudiantes, además, otro tanto utilizó plataformas para la entrega de tareas. Teniendo la ventaja que su comunicación con los estudiantes fue más cercana. (Cardenas, 2020), sin duda los colegios hacen más esfuerzos porque deben responder a la carga económica que conlleva, y han sido más de mil los que cerraron en el año 2020 (Menchú, 2021). 
Siendo el Ministerio de Educación quien a través del Plan integral para la prevención, respuesta y recuperación ante el coronavirus (Covid 19) puso en marcha para la atención de los estudiantes y las directrices para el desarrollo del trabajo en el confinamiento (Ministerio de Educación, 2020).

Un ejemplo de cómo se llevó a cabo el proceso en diversas escuelas, fue, que el Ministerio de Educación distribuyó guías de trabajo académico de acuerdo al grado a las escuelas, las cuales entregaban a los padres de familia una vez por semana y el mismo proceso para devolver los trabajos ya realizados por los niños y de esa forma, ser calificados. Con esta estrategia se logró terminar el año escolar y la promoción de todos los estudiantes que cumplieron con el desarrollo de las guías (Ministerio de Educación, 2020).

Es necesario hacer una evaluación del primero año de educación a distancia y observar si se ha logrado cumplir con las intencionalidades que el Curriculum Nacional Base determina para el alcance de competencias que están estipuladas por curso y que deben ser alcanzadas por los estudiantes en cada grado y estar preparado para el nivel superior (Ministerio de Educación, 2020). Además, se debe recordar que la educación es el bastión para el desarrollo y se convirtió en fundamental para el avance de cualquier país, y que debe procurarse en cualquier situación (Plan Educativo Nacional, 2020).

Unicef realizó un informe en noviembre de 2020, en donde advirtió que los niños son los más afectados en la pandemia, porque son los más vulnerables y los daños pueden llegar a ser irreversibles si no se trabaja en ellos ahora. La educación, es uno de los factores determinantes porque se marca aún más la brecha entre clases sociales y la diferencia traerá repercusiones mayores en más rubros, como nutrición, salud y la economía misma en una generación completa (Unicef, 2020).

En otro de los informes que Unicef ha realizado, determina que la pandemia ha sido devastadora sobre la educación en los países que registran ingresos bajos o medianos bajos porque los recortes de personal, despidos y estancamiento económico vinieron afectar a las familias y a limitar el acceso de educación (Unicef, 2020). Además, el informe también muestra su preocupación en el retorno a clases porque los establecimientos no poseen los requerimientos esenciales 
para los cuidados de los niños con lavado de manos, distanciamiento social y demás requisitos que obligan para mantener la seguridad en salud.

\section{Materiales y métodos}

La investigación fue descriptiva y se utilizó una entrevista para los maestros de instituciones privadas y públicas a nivel primario (30 maestros en total), así como, 20 directores de ambas instituciones. Todos en la ciudad capital de Guatemala.

\section{Resultados}

Después de un año de pandemia y con cambios drásticos en el estilo de educación se hace un análisis de la perspectiva de los maestros, tratando de resumir los aspectos más importantes, además, se hará la comparación entre instituciones privadas y públicas para conocer la forma en que se acercaron a los estudiantes y el seguimiento de la familia para el apoyo en la educación.

La primera pregunta realizada fue el mecanismo que su utilizó para comunicarse con sus estudiantes en el 2020. Las respuestas fueron variadas porque la iniciativa privada utilizó en un $100 \%$ aplicaciones para conferencias como zoom, meet, skipe, entre otras, teniendo una comunicación bastante directa con los estudiantes y al principio de la pandemia, los padres participaban mucho dentro de las reuniones. Pero cuando los adultos responsables de los niños regresaron a sus trabajos, la comunicación quedó asignada a los niños, los más pequeños requirieron un poco más de acompañamiento que los niños más grandes.

Por otro lado, maestros de instituciones públicas, no obtuvieron el acceso en comunicación con los alumnos tan fácilmente, algunos de ellos utilizaron el celular, aplicaciones como WhatsApp, vídeos, llamadas telefónicas, pero esto se limitó porque padres no tenían tanto acceso a internet. El Ministerio de Educación diseñó el Plan integral para la prevención, respuesta y recuperación ante el coronavirus (Covid 19) (Ministerio de Educación, 2020), en donde se trabajó con material impreso para que las escuelas pudieran entregar a los alumnos y los maestros pudieran recibir y calificar. El segundo cuestionamiento fue 
conocer la relación que se mantenía con los niños en el momento de clase. Los maestros de establecimientos privados indicaron que les costaba mucho mantener la atención de los niños, porque se distraían mucho más que estando dentro del aula. Además, que los padres en algún momento comenzaron a interrumpir las clases porque acompañaban a los niños en el proceso.

Por el contrario, los maestros de instituciones públicas, revisaban guías de trabajos y trataban de hacer avances con vídeos de WhatsApp y algunas llamadas telefónicas, pero los resultados del desarrollo de las guías consideran que no fueron suficientes para determinar si realmente aprendieron.

Y la tercera pregunta fue si consideran si los niños alcanzaron las competencias del año 2020 y si estaban preparados para el próximo nivel académico. $Y$ el grupo completo de maestros manifestaron que son muy pocos los niños que alcanzaron las competencias del grado que desarrollaron. Es importante acotar que los maestros de nivel privado fueron más optimistas en sus respuestas que los maestros de las instituciones públicas.

Con respecto a la entrevista realizada a directores de establecimientos públicos y privados, se expone el resultado a continuación:

Se considera al padre de familia como parte fundamental en el acompañamiento en el proceso de educación en tiempos de pandemia. Los padres de niños de iniciativa privada trataron de involucrarse en la educación de los mismos, al principio fueron muy críticos y no entendían mucho las dinámicas utilizadas por los maestros y enfrentarlos a tecnología fue parte de ese reto.

Los directores de las escuelas públicas, manifestaron la preocupación que los padres sintieron al principio de la pandemia y muchos de ellos con buenas intenciones, pero sin el conocimiento no podían apoyar mucho a sus niños. Y la segunda preocupación que abordó a los padres fue la económica porque muchos de ellos se quedaron sin trabajo y aunque la educación es gratuita siempre necesitan de ciertos recursos para el desarrollo del proceso educativo, aunque las bolsas de alimentos fueron una buena estrategia para que los niños no abandonaran la escuela y que la deserción escolar fuera mayor. 
Por último, la preocupación que los directores manifestaron fue que al crear las condiciones de la educación virtual o a distancia, hizo que lo contenidos no fueran desarrollados de manera óptima y que las competencias no fueran alcanzadas satisfactoriamente. Consideran que los niños aprendieron, pero no al nivel de cuando el proceso ha sido presencial.

\section{Discusión}

Un año después del inicio de la pandemia y con un año escolar concluido e iniciado el nuevo ciclo escolar, la experiencia de maestros y directores ha sido vertiginoso porque tuvieron muchos cambios, ya que comenzaron presencial y terminaron virtual, muchos de ellos, sin tener un conocimiento profundos en tecnología (Sánchez-Villena, \& De La Fuente-Figuerola, 2020).

El alcanzar las competencias que los estudiantes deben desarrollar en cada proceso académico es fundamental para poder enfrentar el nuevo nivel. Tanto maestros y directores manifestaron que dentro de un aula es mucho más sencillo captar la atención, identificar los niveles de aprendizaje que poseen los niños y poder retroalimentar de manera personalizada era mucho más sencillo. Contrario a lo sucedido con educación a distancia, porque padres, niños y maestros tuvieron que converger en tiempos limitados en un ambiente nuevo y con desafíos de tecnología, en el más favorable de los casos.

De acuerdo a las exposiciones de expertos en CNN Mundo, el proceso de educación presencial no podrá ser sustituida por la educación virtual, la interacción entre maestros y alumnos, es fundamental para el desarrollo de los niños dentro del proceso de educación, porque la interacción social es fundamental para el proceso de aprendizaje. (CNN MUNDO, 2020), aunque por los tiempos que vivimos la virtualidad es un proceso que llego y se quedará (Diálogos Social).

En uno de los informes que Unicef ha presentado, manifiesta en uno de sus informes que la pandemia ha venido afectar directamente a los niños en países en vías de desarrollo y que se tendrá una generación que perderá mucho en más que un año escolar, porque la disparidad de oportunidades del acceso a la educación a distancia se incremetarán en problemas de desarrollo económico, en salud y por supuesto, la educación (Unicef, 2020). 
Por medio de la entrevista, los maestros expresaron las dificultades con respecto a comunicación que tuvo con los estudiantes y se evidenció claramente la diferencia entre instituciones públicas y privadas y como la tecnología apoyó a un grupo, pero no a otro, abriendo brecha entre las clases que convierte a la educación en diferencia que marca más la desigualdad.

El Curriculum Nacional Base sienta los fundamentos de las competencias que cada grado debe alcanzar para que los niños tengan la oportunidad de enfrentar los nuevos retos del nivel superior. Con todo lo vivido por la pandemia en el 2020, la oportunidad a los estudiantes de llevar las clases a distancia, ofreció la ventaja de aprobar un grado, pero el conocimiento y las competencias que se debían alcanzar, no fueron logradas del todo, porque los niños no desarrollaron todas las capacidades esperadas en los lineamientos de cada grado.

Los maestros y directores manifiestan que los resultados que se tienen niveles de aprendizaje del año pasado para este 2021 son bajos y que este nuevo ciclo tendrá muchas más demandas que las que tuvo el año anterior. $Y$ que se deben buscar estrategias para en un futuro con clases presenciales o un proceso híbrido de educación se puedan tratar de alcanzar. Además, que el contacto humano es fundamental para el desarrollo integral de los niños, no importando su edad.

Para países en desarrollo como Guatemala, el regreso a clases presenciales es necesario, en un proceso normal o híbrido para poder nivelar a los estudiantes, y también estar conscientes que la salud y la vida son fundamentales. Por lo que el futuro depara, (Durán Rodríguez, 2015), será un proceso muy arduo y de mucho compromiso de parte de las instituciones educativas representadas por los directores y maestros, de los padres y de los mismos estudiantes para minimizar esta brecha de desarrollo que se cimienta en la educación.

\section{Agradecimientos}

A maestros y directores de colegios privados y escuelas públicas ubicados en la zona 1, 2 y 7. También a la Dra. Maribel Valenzuela catedrática y coordinadora de Postgrado en la Facultad de Humanidades de la Universidad de San Carlos de Guatemala. Y a la MSc. Victoria Contreras, como asesora de estilo. 


\section{Referencias}

Agenda Guatemalteca de Noticias. (2021). Covid 19: Colegios toman medidas ante modificación del tablero de alertas sanitarias que emitió salud. Obtenido de https://agn.gt/covid-19-colegiostoman-medidas-ante-modificacion-de-tablero-de-alertasanitaria-que-emitio-salud/

Cardenas, J. (2020). La educación privada se blinda con más normas que las indicadas por las comunidades. agosto. Obtenido de https://cincodias.elpais.com/cincodias/2020/08/19/ economia/1597857076_743741.html

CNN MUNDO. (2020). Las ventajas y desventajas de la educación virtual. CNN MUNDO. Obtenido de https://cnnespanol.cnn. com/2020/12/23/de-la-educacion-presencial-a-la-virtual-losdesafios-para-los-estudiantes-debido-a-la-pandemia/

Diálogos Social. (2020). La virtualidad llegó para quedarse. La virtualidad Ilegó para quedarse vídeo. Obtenido de https:// youtu.be/iuirVSai_vl

Durán Rodríguez, R. (2015). La Educación Virtual Universitaria como medio para mejorar las competencias genéricas y los aprendizajes a traves de buenas prácticas docentes. Tesis doctoral. Barcelona. Obtenido de https://www. tdx.cat/bitstream/handle/10803/397710/TRADR1de1. pdf?sequence $=1$ \&isAllowed $=y$

Menchú, S. (01 de febrero de 2021). Mil colegios en quiebra por la pandemia. No ficción. Obtenido de https://www.no-ficcion. com/project/mil-colegios-quiebra-pandemia

Ministerio de Educación. (25 de mayo de 2020). Gobierno de Guatemala. Obtenido de Ministerio de Educación: http://www.mineduc. gob.gt/portal/orientaciones-para-trabajar-en-casa.html

Ministerio de Educación. (2020). Plan integral para la prevención, respuesta y recuperacióbn ante el coronavirus (Covid 19). Guatemala. Obtenido de https://www.mineduc.gob.gt/ portal/documents/PLAN-DE-RESPUESTA-CORONAVIRUSCOVID-19-010620.pdf 
Ola, A. L. (30 de marzo de 2020). Presna Libre. Obtenido de Coronavirus:educación pública tiene el desafío de recuperar clases por suspensión: https://www.prensalibre.com/ guatemala/comunitario/coronavirus-educacion-publica-tieneel-desafio-de-recuperar-clases-por-suspension/

Organización Mundial de la Salud. (25 de mayo de 2020). Organización Munidal de la Salud. Obtenido de Organización Mundial de la Salud: https://www.who.int/es/emergencies/diseases/novelcoronavirus-2019?gclid=CjwKCAjw2a32BRBXEiwAUcugiJmQT5 mqZQ-9znNLOi1kGvs-qCOi7Nf4glhcv3Bt5etpaROOVURERBoC 7wWQAvD_BwE

Plan Educativo Nacional. (25 de mayo de 2020). Hacia la reforma del Sistema Educativo Nacional. Obtenido de Hacia la reforma del Sistema Educativo Nacional: http://www. planeducativonacional.unam.mx/CAP_00/Text/00_05a.html

Sánchez-Villena, \& De La Fuente-Figuerola. (5 de junio de 2020). COVID-19: cuarentena, aislamiento, distanciamiento social y confinamiento ¿son lo mismo? Anales de Pediatría. doi: 10.1016/j.anpedi.2020.05.001

Unicef. (2020). Los niños de los países más pobres han perdido casi cuatro meses de escolarización desde que comenzó la pandemia, según la UNESCO, UNICEF y el Banco Mundial. Nueva York. Obtenido de https://www.unicef.org/guatemala/ comunicados-prensa/los-ni\%C3\%B1os-de-los-pa\%C3\%ADsesm\%C3\%A1s-pobres-han-perdido-casi-cuatro-meses-de

Unicef. (2020). UNICEF pide evitar una generación perdida a medida que la COVID-19 amenaza con causar daños irreversibles a la educación, la nutrición y el bienestar de los niños. Obtenido de UNICEF pide evitar una generación perdida a medida que la COVID-19 amenaza con causar daños irreversibles a la educación, la nutrición y el bienestar de los niños 
Facultad de Humanidades Escuela de Estudios de Postgrado

\section{Sobre la autora \\ Es Licenciada en Administración de Empresas, con una maestría en ciencias en Innovación Educativa y otra en Educación con especialidad en Educación Superior. Además, estudiante del Doctorado en Educación de la Universidad San Carlos de Guatemala.}

Copyright (c) Carmen María Santiso Rodríguez

\section{(c) (i) \\ BY}

Este texto está protegido por una licencia Creative Commons 4.0.

Usted es libre para compartir, copiar y redistribuir el material en cualquier medio o formato y adaptar el documento, remezclar, transformar y crear a partir del material para cualquier propósito, incluso comercialmente, siempre que cumpla la condición de atribución: usted debe reconocer el crédito de una obra de manera adecuada, proporcionar un enlace a la licencia, e indicar si se han realizado cambios. Puede hacerlo en cualquier forma razonable, pero no de forma tal que sugiera que tiene el apoyo del licenciante o lo recibe por el uso que hace. 